\title{
micromachines
}

ISSN 2072-666X

www.mdpi.com/journal/micromachines

Article

\section{Passive Micromixers with Interlocking Semi-Circle and Omega-Shaped Modules: Experiments and Simulations}

\author{
Ala'aldeen Al-Halhouli 1,2,*, Aiman Alshare ${ }^{3}$, Mukeet Mohsen ${ }^{1}$, Maher Matar ${ }^{\text {, }}$ \\ Andreas Dietzel ${ }^{1}$ and Stephanus Büttgenbach ${ }^{1}$
}

1 Institute of Microtechnology, Technical University of Braunschweig, Braunschweig 38106, Germany; E-Mails: mukeet2004@yahoo.de (M.M.); m.matar@tu-braunschweig.de (M.M.); a.dietzel@tu-braunschweig.de (A.D.); s.buettgenbach@tu-bs.de (S.B.)

2 Mechatronics Engineering Department, German Jordanian University, Amman 11180, Jordan

3 Mechanical Engineering Department, German Jordanian University, Amman 11180, Jordan; E-Mail: aiman.share@gju.edu.jo

* Author to whom correspondence should be addressed; E-Mail: alaaldeen.alhalhoul@gju.edu.jo; Tel.: +962-642-945-11; Fax: +962-643-002-15.

Academic Editor: Jeong-Bong Lee

Received: 3 June 2015 / Accepted: 14 July 2015 / Published: 22 July 2015

\begin{abstract}
This study presents experiments and computational simulations of single-layer passive micromixer designs. The proposed designs consist of chains of interlocking semicircles and omega-shaped mixing modules. The performance of the new designs is compared with the concentric spiral channel configuration. The micromixers are intended to be integrated into a lab on chip (LOC) micro-system that operates under continuous flow conditions. The purpose behind the multi-curvature in these designs is the introduction of Dean vortices in addition to molecular diffusion in order to enhance the mixing performance. The micromixers were fabricated in PDMS (Polydimethylsiloxane) and bonded to a glass substrate. A three-dimensional computational model of micromixers was carried out using Fluent ANSYS. In experiments, the mixing of a $1 \mathrm{~g} / \mathrm{L}$ fluorescein isothiocyanate diluted in distilled water was observed and photographed using a charge-coupled device (CCD) microscopic camera. The obtained images were processed to determine the mixing intensity at different Reynolds numbers. The standard deviation $(\sigma)$ of the fluorescence indicates the mixing completeness, which was calculated along the width of the channel at various locations downstream from the channel inlet. The value of $\sigma=0.5$ indicates unmixed streams and 0 is for complete mixing. It is found that the two new designs have a standard deviation of nearly 0.05 . Additionally, complete mixing was observed at the channel outlet as demonstrated by
\end{abstract}


the fluorescence images and the numerical results. However, the location of complete mixing at different positions depends on the Reynolds number, which varies between 0.01 and 50 . Good agreement was found between the experiment and the numerical results. A correlation to predict the length scale where complete mixing can be achieved is given in terms of the radius of curvature, the mixing module, and the Reynolds number.

Keywords: passive micromixers; dean vortices; Reynolds number; microfluidics; LOC

\section{Introduction}

Due to the rapid increase in demanding biomedical/biotechnological applications, continuous efforts have been directed towards developing novel and efficient microfluidic devices (i.e., pumps, sensors, mixers, and bioreactors) [1-3]. The flow in such devices is mainly laminar [4]. Under such conditions, turbulence eddies are missing and rapid mixing is inefficient because the main mixing mechanism is diffusion and the intermolecular diffusion of macromolecules is too slow to cause effective mixing at small time scales. Accordingly, the research on developing rapid micromixers increases and several passive and active micromixer designs have been investigated recently [5-8]. These micromixers are inspired by advancements and lofty requirements of, e.g., drug delivery, biological processes, and lab-on-a-chip (LOC) platforms for biochemical analysis.

Active mixers employ external forces (e.g., electro-osmosis, magnetic-stirring, and ultrasonic) to perform mixing. These designs often add complexity to the microfluidic chip fabrication process because the external actuator should be integrated into the system [9]. In contrast, passive mixers may be easily fabricated as part of the microfluidic chip since they do not require external forces to operate. However, such devices require longer mixing length in order to achieve uniform and complete mixing [10].

To reduce the mixing lengths in passive micromixers, different designs based on chaotic advection, lamination, and Dean vortices have been developed [7-9]. Chaotic advection refers to producing chaotic particle trajectories in a simple regular velocity field. This can occur when a velocity field is either two-dimensional and time-dependent or three-dimensional with or without time dependence. This typically indicates rapid distortion and elongation of flowing material interfaces, which accordingly increases the area across which diffusion occurs and leads to rapid mixing. This effect was successfully implemented in a passive micromixer of a three-dimensional serpentine channel with "C-shaped" repeating units. To realize a three-dimensional (3D) channel geometry, the device was fabricated in a silicon wafer using a double-sided $\mathrm{KOH}$ wet-etching technique [11]. Experiments on the "C-shaped" serpentine channel showed that it is superior to the square-wave and the straight channel in terms of fast mixing for Reynolds numbers 6 to 70, and that the serpentine channel mixing capability increases with increasing Reynolds number.

In general, fast mixing can also be achieved with smaller mixing path and larger interface surface. However, this influences the desired high throughput and the high driving pressures [4]. Therefore, two basic lamination concepts for decreasing the mixing path were introduced, namely: parallel lamination and serial lamination. Parallel lamination splits the inlet streams into multiple narrow sub-streams then joins them into one stream as laminae, while in serial lamination the inlet streams are joined/split 
horizontally or vertically in several stages until liquid layers are laminated [5]. This concept was tested in making controlled lamination in a plane channel system with patterned grooves. The basic idea is that the liquids entering the inlet are exposed to a controlled $90^{\circ}$ rotation of a flow cross-section followed by a split into several channels; the flow in each of these channels is rotated a further $90^{\circ}$ before recombining. This doubles the interfacial area between the two flows. This process is repeated until the desired degree of mixing is achieved. Simulations showed that lamination took place for Reynolds numbers up to 5. It was also proven, using simulations and experimental prototypes, that the combination of patterning the channel bed and splitting and recombining the streams can be used to achieve controlled lamination in a 2D channel system [7].

Micro-mixing by Dean flows mainly depends on recirculating the flow perpendicular to the main flow direction via centrifugal effects experienced by fluids traveling along a curved path. Under appropriate flow and geometrical conditions, Dean flows are induced to increase diffusive transport and promote enhanced mixing in considerably shorter downstream distances as compared to planar straight channels [9]. Mixing in spiral channels was tested using five different designs of multiple sections for Reynolds numbers between 0.02 and 18.6 [9]. Results showed mixing levels of more than 95\% in the eight-arc and ten-arc channels. The mixing efficiency with Dean vortex-based designs demonstrated improvement using two-dimensional (2D) passive planar labyrinth of S-shaped geometry. Results showed that the uniform and fast mixing can be improved by adding a short straight channel between two consecutive semicircles arranged with a $180^{\circ}$ turn for Reynolds numbers between 2.5 and 30 [8].

Experiments and simulations of passive mixing using (2D) modified Tesla structures of a microchannel were carried out; one of the fluids is divided into two sub-streams and then merged again with the other fluid from the main channel of the micromixer. Then they were mixed with the other sub-stream, producing a strong impact around the sub-channel of the micromixer by exploiting the Coanda effect [12]. The splitting and recombining of the flow effectively reduce the diffusion path between the fluid streams by producing transverse Taylor dispersion, which enhances the convective mixing of the two fluids [12]. The performance of the mixer is tested with flows of $1 \mu \mathrm{L} / \mathrm{min}$ to $100 \mu \mathrm{L} / \mathrm{min}$; it gave an excellent mixing level of $44 \%$ concentration and a reasonable agreement was found between the experiments and the CFD results. In order to further enlarge the contact surface formed by two or more sub-streams in multi-layer microfluidic channels and to further enhance the strength of mixing, 3D Tesla structures are investigated experimentally and by CFD analysis [13]. The steady 3D computational model of mass, momentum, and species concentration distribution results were compared with fluorescence images of concentration; the investigated range of flow rate was $0.015 \mu \mathrm{L} / \mathrm{s}$ to $15 \mu \mathrm{L} / \mathrm{s}$, corresponding to Reynolds numbers of 0.1-100. The proposed 3D Tesla micromixer was utilized to conduct immunofluorescence experiments of the H1975 cancer cells conjugated with rabbit anti-EGFR L858R antibodies, revealing the mixing effectiveness of the micromixer in the binding reaction of antibodies for detecting antigens of lung cancer cellular surface [13].

The current study presents the experimental and computational simulations performed on various single layer passive micromixers composed of mixing units shaped as spiral, interlocking-semicircle, and $\Omega$ channel designs. Results shows improved mixing for a wide range of Reynolds numbers between 0.01 and 50 . The developed and tested designs have a small footprint and can be easily integrated into a LOC system that requires simple planar geometries. 


\section{Numerical Simulations}

The micromixer's three-dimensional geometry mesh is shown in Figure 1. The problem setup and solver were performed using the commercial software package Fluent ANSYS 14. The 3D model was generated by extruding a 2D design to form a channel $230 \mu \mathrm{m}$ in depth and $200 \mu \mathrm{m}$ in width. The mesh was chosen to be relatively fine with 160,000 elements for the spiral design, 380,000 for the interlocking semicircle design, and 465,000 for the $\Omega$ design. Due to the physics of the problem and its continuous flow operation, a steady state flow condition was assumed. The problem setup includes the solution of Navier-Stokes equations, and the species transport equation for the viscous and laminar flow conditions. Water with a constant diffusion coefficient of $1 \times 10^{-10} \mathrm{~m}^{2} / \mathrm{s}$ was chosen as the working fluid.

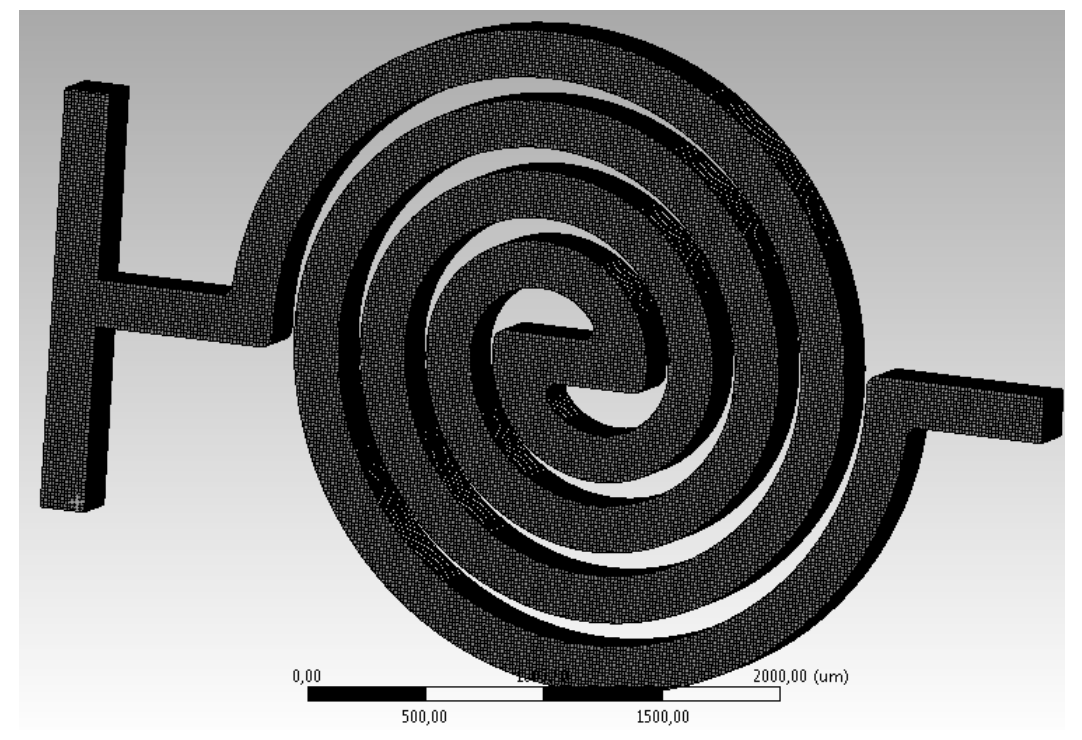

Figure 1. Presentation of the 3D spiral mixer numerical model. Channel depth of $230 \mu \mathrm{m}$ and width of $200 \mu \mathrm{m}$.

The SIMPLEC (SIMPLE-Consistent) scheme [14] is a pressure-based segregated algorithm that was selected for pressure-velocity coupling. It has the same steps as the SIMPLE algorithm [15], with the difference that the momentum equations are manipulated so that the SIMPLEC velocity correction equations omit terms that are less significant than those omitted in SIMPLE. The second order spatial discretization was implemented for the pressure and momentum equations. The QUICK scheme [16] was employed for species diffusion. QUICK is typically more accurate on structured grids aligned with the flow direction, where the second order discretization method was used to obtain accurate results and minimize numerical diffusion encountered in such a complex flow path.

The computational simulations were carried out iteratively and the iterations were stopped when the continuity, velocity components, and species-scaled residual reached a value of $10^{-6}$ or until it stabilized at a constant value that is still small enough to ensure convergence. Furthermore, the mass imbalance was monitored from the flux option, to ensure that it is a small value compared to the total outlet mass flow rate. For example, the mass imbalances in $\Omega$ micromixers at $\mathrm{Re}=10$ and 50 were reported as $4.27 \times 10^{-11} \mathrm{~kg} / \mathrm{s}$ at $\mathrm{Re}=50$ with total inlet mass flow rate of $10.8 \times 10^{-6} \mathrm{~kg} / \mathrm{s}$, while it was $9.89 \times 10^{-12}$ at $\operatorname{Re}=10$ at total inlet mass flow rate of $2.15 \times 10^{-6} \mathrm{~kg} / \mathrm{s}$. These imbalance values are very small compared to the inlet mass flow rates. 
The mixing performance in spiral, interlocking semicircle, and $\Omega$ micromixers was simulated as explained in the following sections. Complete mixing is considered to be when mass fraction numerical results of $45 \%$ or greater are achieved.

\subsection{Spiral Design}

Spiral micromixers with satisfactory results in very small mixing channel widths of approximately $29 \mu \mathrm{m}$ have been studied previously [9]. In the present work, a spiral micromixer with channel widths of $200 \mu \mathrm{m}$ was designed, simulated, and experimentally tested. Since the mixing in the microfluidic systems takes place mainly by molecular diffusion, the mixing channel length and cross section play a crucial role on the mixing performance. However, the spiral channel geometry offers additional passive mixing possibility through the Dean flow. Dean vortices are a result of the variation in centrifugal forces due to the velocity differences within the channel cross-section, which result in a secondary flow perpendicular to the main flow direction. The magnitude of these secondary flows is measured by Dean number $\left(D e=\operatorname{Re} \sqrt{D_{h} / 2 R}\right.$ ), which is affected by the flow velocity (i.e., Reynolds number, Re), the hydraulic diameter $D_{h}$, and channel radius of curvature $R$. The strength of the Dean vortices increases with increasing velocity and decreases with increasing radius of curvature, as illustrated in Figure 2.

As a result of centrifugal acceleration in the radial direction, two symmetrical counter-rotating vortices are formed in a given channel cross section (top and bottom). This lateral motion translates the liquids between the inner and outer walls, which increases the fluid's interfacial area along channel width and accordingly enhances the mixing performance. Calculated local Dean number as a function of the Reynolds number is given in Table 1.

(a)

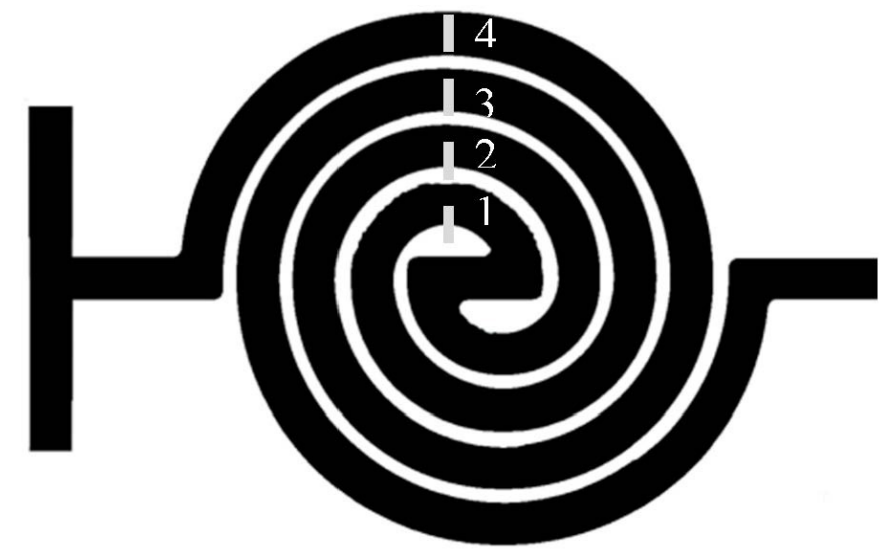

(b)

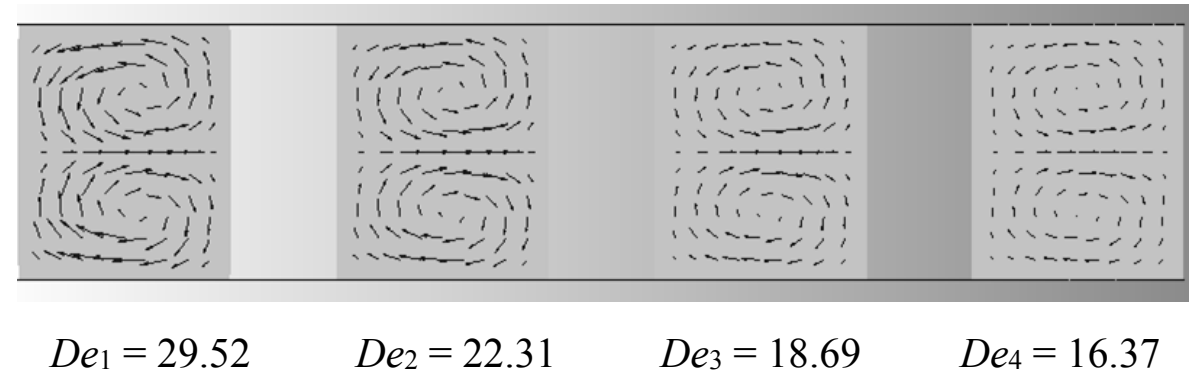

Figure 2. (a) Two-dimensional schematic of the spiral micromixer and (b) plots of generated counter rotating Dean vortices vectors at $\mathrm{Re}=50$ for cross sections $1,2,3$, and 4 (The longer the arrows, the stronger the vortex rotational speed). 
Table 1. Calculated Dean number at spiral channel sections 1 to 4 and Reynolds numbers.

\begin{tabular}{ccccc}
\hline $\boldsymbol{R} \boldsymbol{e}$ & $\mathbf{0 . 0 5}$ & $\mathbf{1 . 0}$ & $\mathbf{5}$ & $\mathbf{5 0}$ \\
\hline Section No. & \multicolumn{5}{c}{ Dean Number, De } \\
\hline 1 & 0.0295 & 0.5903 & 2.95 & 29.52 \\
2 & 0.0223 & 0.4463 & 2.23 & 22.31 \\
3 & 0.0187 & 0.3734 & 1.87 & 18.69 \\
4 & 0.0164 & 0.3275 & 1.64 & 16.37 \\
\hline
\end{tabular}

\subsection{The Interlocked Semi-Circle (ILSC) and Omega ( $(\Omega)$ Designs}

Inspired by mixing simulation results for the spirals, two new micro-mixer designs are proposed to exploit the favorable influence of Dean vortices on the mixer performance. The first shape was arranged using a series of interlocking-semicircle mixing modules with a $300 \mu \mathrm{m}$ mean radius. Each mixing module or mixing unit is composed of two offset mirrored interlocking semi-circles (ILSC) as shown in Figure 3a. This configuration is expected to yield stronger Dean vortices along the channel at the same Reynolds number as the spiral design. The micro-mixer channel total length is $54 \mathrm{~mm}$.

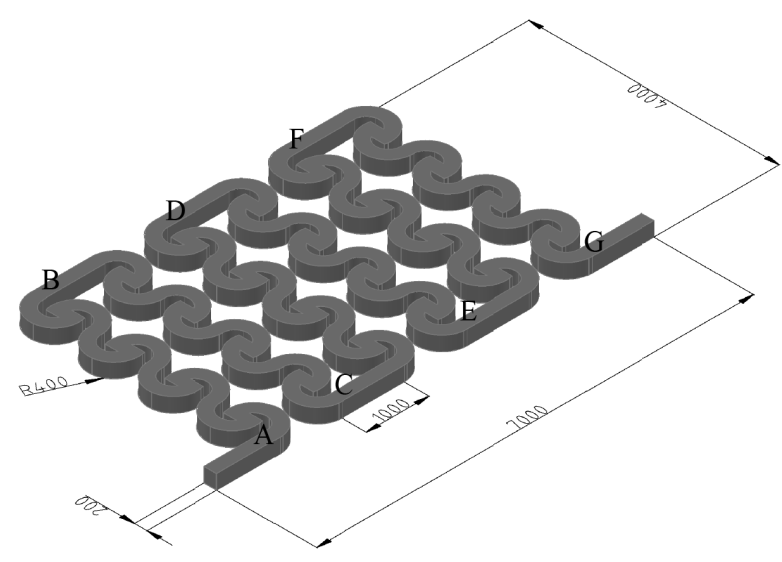

(a)

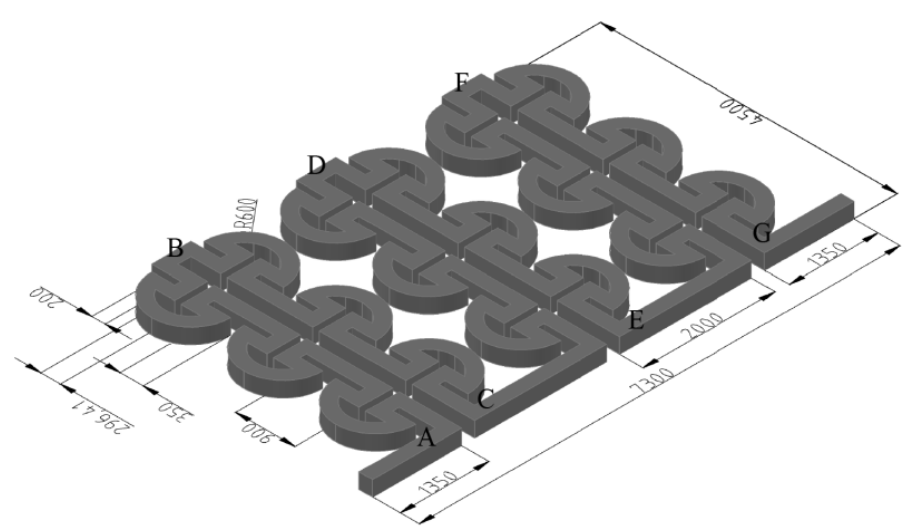

(b)

Figure 3. Dimensioned geometries of the (a) ILSC and (b) $\Omega$ mixers.

The ILSC arrangement enables the flow to change its direction within each mixing module and accordingly changes the Dean vortices' direction, as illustrated in Figure 4 by the two selected cross-stream sections showing the velocity vector plots superimposed on the mixing intensity counters. This change in flow direction is particularly advantageous at higher Reynolds numbers, because the Dean number is directly proportioal to the Reynolds number, and the increase in flow inertia translates into increase in vorticity, hence, enhanced mixing.

The second design is a series of Omega $(\Omega)$-shaped modules. This configuration enables a simultaneous rapid flow direction change in the $\Omega$-shaped channel, as shown in Figure $3 \mathrm{~b}$. The flow changes its direction four and six times by $90^{\circ}$ within each $\Omega$ mixing module and ILSC module, respectively, as shown in Figure 5a,b. The mean radius of curvature of the $\Omega$ and ILSC designs is $500 \mu \mathrm{m}$ and $300 \mu \mathrm{m}$, respectively. Therefore, it is expected that the strength of Dean vortices in the $\Omega$ design would be less than that in the ILSC design for a given Reynolds number. 

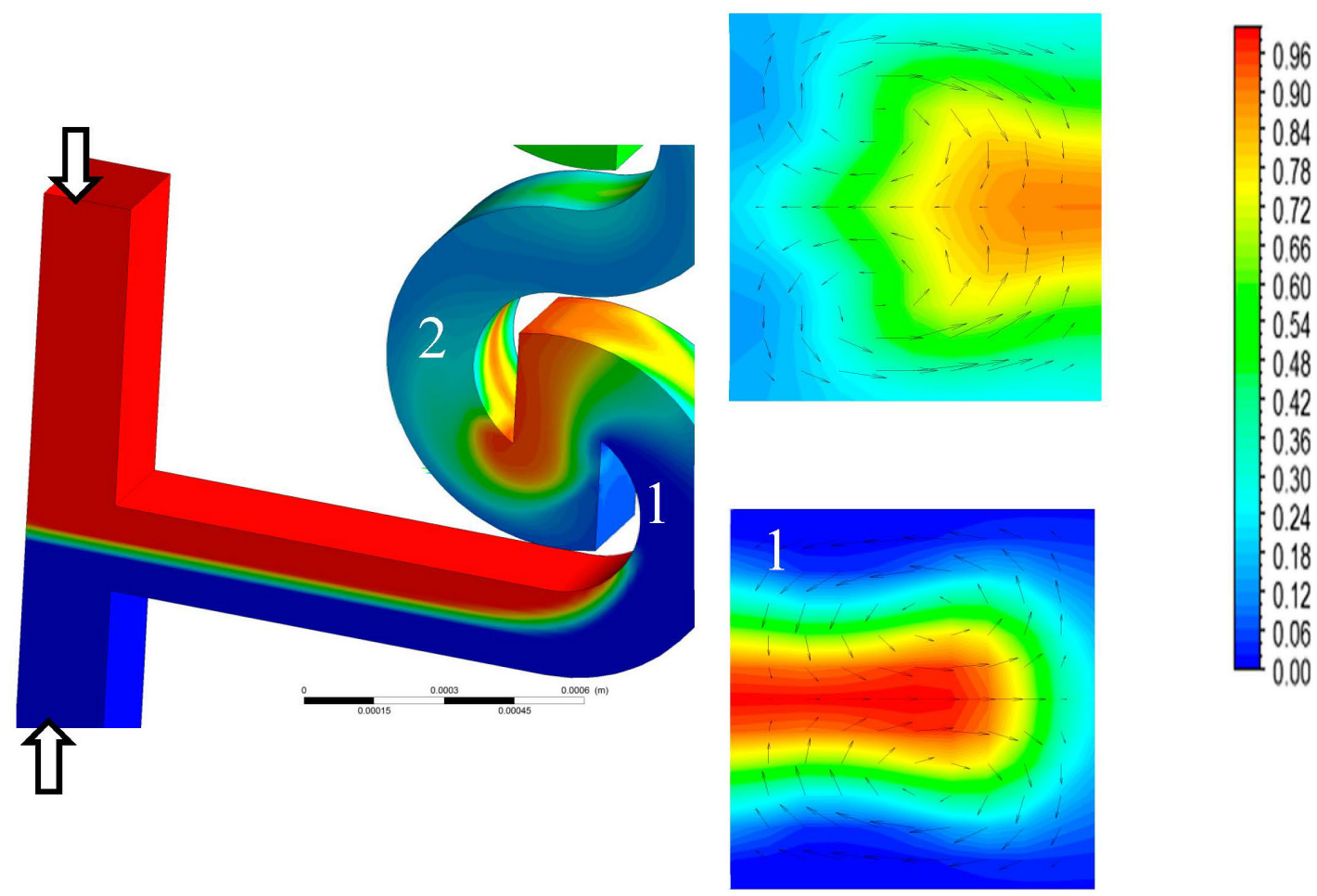

Figure 4. Dean vortices at $\operatorname{Re}=50$ at sections 1 and 2 of the first semicircle-mixing unit.

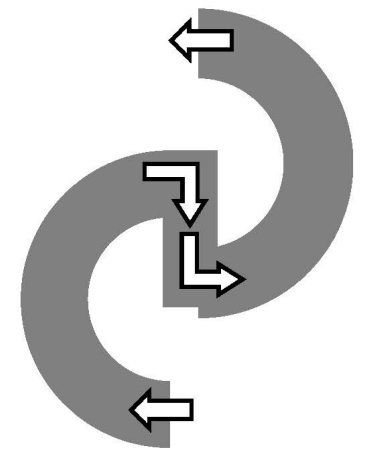

(a)

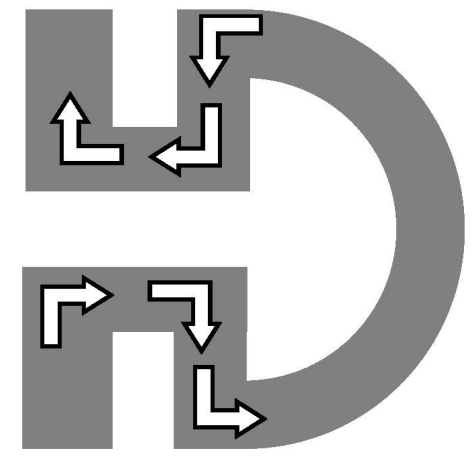

(b)

Figure 5. Rapid flow reversal in (a) ILSC mixing module and (b) rapid flow deflection in the $\Omega$ mixing module.

The simulation results of the mass fraction contours for both designs at different Reynolds numbers $(\operatorname{Re}=0.01,0.1,1,5,10,20,30,40,50)$ are shown in Figure 6. Apparently, the number of required modules to achieve full mixing decreases with increasing Reynolds number, which results in increasing dean flow.

The local mass fractions for the ILSC and $\Omega$ channels are shown in Figure 6a,b, respectively. It is interesting to note that by increasing the Reynolds number, the Dean flow comes into effect and dominates the molecular diffusion effect, practically reducing the number of modules required to achieve complete mixing.

The mass fraction percentage data of mixing for the two simulated designs are plotted for a wide range of Reynolds numbers (0.01-50) as displayed in Figure 7. 

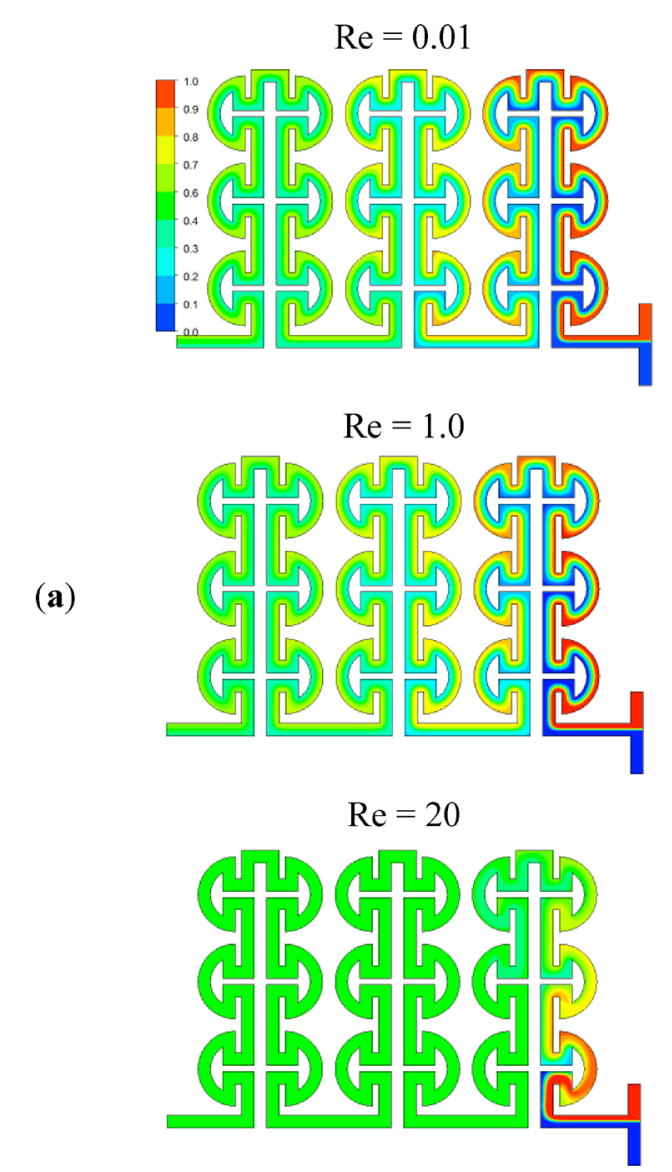

$\mathrm{Re}=0.01$

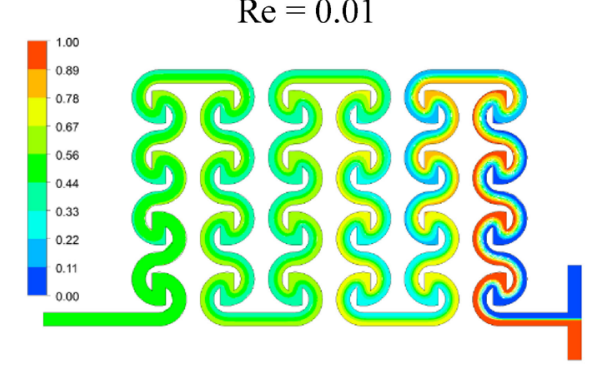

$\operatorname{Re}=1.0$

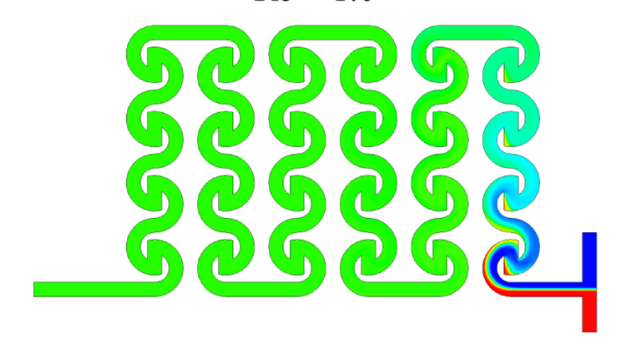

$\mathrm{Re}=20$

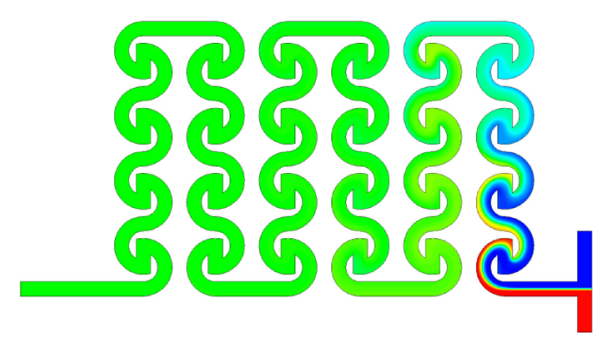

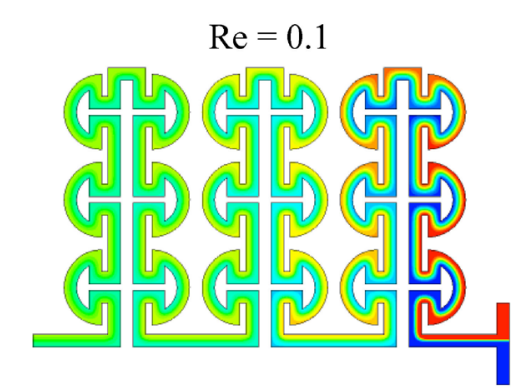

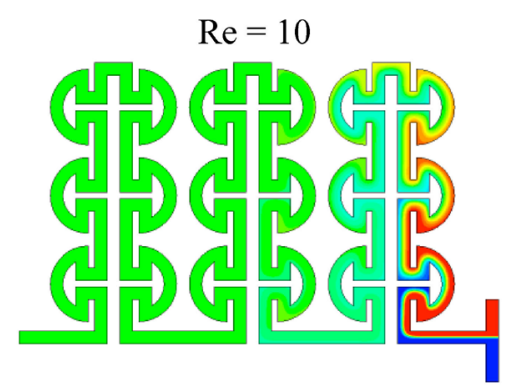

$\mathrm{Re}=50$

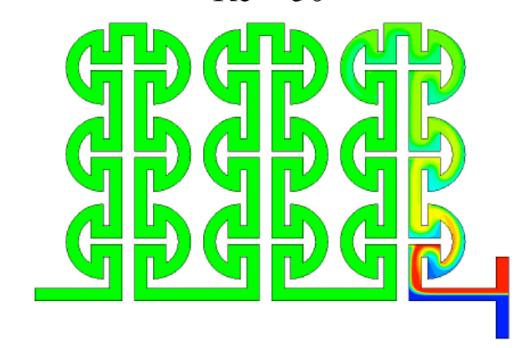

$\operatorname{Re}=0.1$

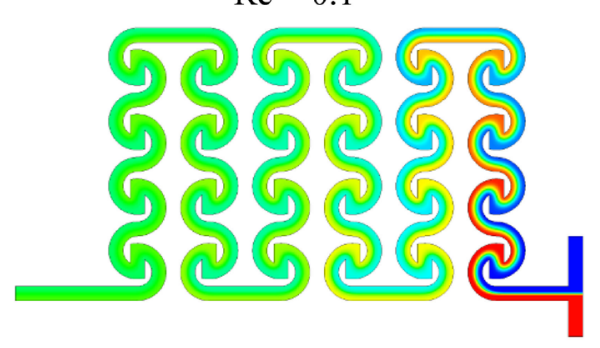

$\mathrm{Re}=10$

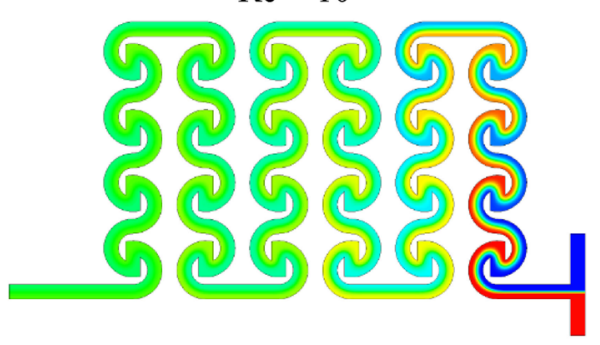

$\mathrm{Re}=50$

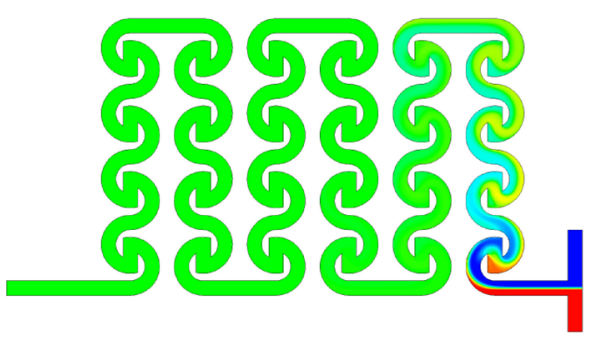

Figure 6. The species mass fraction contours taking at a mid-plane in the (a) $\Omega$ micro-channel and (b) ILSC micro-channel. Sequence of increasing Reynolds number: 0.01, 0.1, 1, 10, 20, 50. 


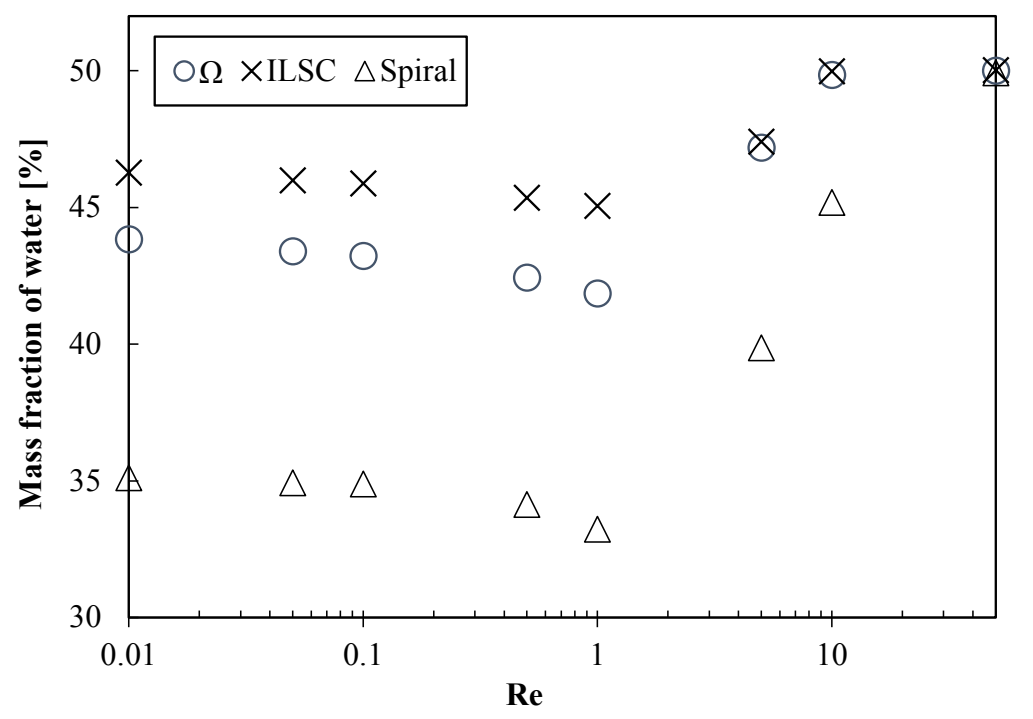

Figure 7. Mass fraction of water species at the outlet for different micromixers.

At low Reynolds number in the range $\mathrm{Re}<1$, the mixing of two fluids is low and predominantly depends on the diffusion of fluid particles through the entire cross section of the channel; accordingly extremely long channels with small characteristic dimensions are required. Figure 7 shows that the eight-arc spiral design as a base case to compare the two newly proposed designs. The spiral channel has a width of $200 \mu \mathrm{m}$ and a length of about $22 \mathrm{~mm}$. The simple spiral channel has the worst mixing performance at all Reynolds numbers. However, constructing the micro-channel of mixing modules such as ILSC and $\Omega$ not only improves the mixing performance but also reduces the footprint length required for the often compact LOC applications. It is interesting to note, as shown in Figure 7, that when increasing the Reynolds number $(\operatorname{Re} \rightarrow 1)$, the mixing performance decreases slightly with increasing flow velocity. This can be related to the decrease of the fluid particles' residence time as well as the weak Dean vortices effect on mixing. Thereby, the fluid particles do not have sufficient time for diffusion through the entire channel cross section. Increasing the flow velocity further $(\operatorname{Re}>1)$, the effect of Dean vortices and inertial forces becomes noticeable and a rapid enhancement on the mixing performance is observed for both of the micromixer designs. A complete mixing is achieved at $\operatorname{Re}>10$ for the ILSC and $\Omega$ (Figure 7). The ILSC micromixer appears to be superior and provides complete mixing over the entire Reynolds number range considered in this study. The results of micromixers are compiled in terms of the traversed length where complete mixing occurs and the radius of the curvature that characterizes the mixing module; they are correlated in terms of the Reynolds number. The proposed correlation (Equation (1)) is as follows:

$$
\frac{L_{\text {mix }}}{R}=\frac{1}{c_{1}+c_{2} R e_{D_{H}}}
$$

where the constants $c_{1}$ and $c_{2}$ are 0.0106 and 0.0158 , respectively.

\subsection{Materials and Fabrication}

The photoresists SU-8 2005 and SU-50 and their development solution, propylene glycol methyl ether acetate (PGMEA), were purchased from MicroChem Corporation (Newton, MA, USA). The PDMS 
Sylgard 184 elastomer kit was bought from Dow Corning (Midland, MI, USA) and was used according to the datasheet.

The micromixers are fabricated by applying UV-depth lithography for the fabrication of the SU-8 negative master and soft lithography processing for PDMS molding. The master is prepared by patterning a single-layer photolithographic process onto a glass substrate. The fabrication process begins with cleaning and dehydrating the substrate on a hotplate for $1 \mathrm{~h}$ at $120^{\circ} \mathrm{C}$. After that, a photolithographic process starts with spinning on a thin layer of SU-8 2005 on the substrate at $2500 \mathrm{rpm}$ for $30 \mathrm{~s}$ and subsequently drying it for $10 \mathrm{~min}$ at $100{ }^{\circ} \mathrm{C}$. The SU-8 layer is then flood exposed to UV light with $100 \mathrm{~mJ} / \mathrm{cm}^{2}$ and baked at $95{ }^{\circ} \mathrm{C}$ for $20 \mathrm{~min}$. This layer is about $5 \mu \mathrm{m}$ in thickness and acts as an adhesion promoter and seed layer for the next structure.

The micromixer's body is then patterned by processing a single layer of SU-8 50. Four milliliters of the photoresist is spun at $600 \mathrm{rpm}$, leveled at planar plate, and then dried at $100{ }^{\circ} \mathrm{C}$. A layer thickness of $230 \mu \mathrm{m}$ is obtained after this processing step. After that, the substrate is exposed to UV light at $650 \mathrm{~mJ} / \mathrm{cm}^{2}$ and baked for $20 \mathrm{~min}$ at $95^{\circ} \mathrm{C}$. The exposed master is then left overnight and developed the next day in PGMEA.

A replica of this master is formed by using PDMS. The PDMS pre-polymer is prepared by mixing the curing agent with elastomer base in a 1:10 ratio (volume:volume). The mixture is then degassed to remove air bubbles, poured onto the master, heated at $70{ }^{\circ} \mathrm{C}$ for $60 \mathrm{~min}$, and finally peeled off from the master. PDMS micromixer molds are then bonded to glass chips and connected with inlet and outlet ports. Pictures of both fabricated micromixers are shown in Figure 8.

A syringe pump (Fusion 100, KR Analytical Ltd., Cheshire, UK), USB digital microscope 1.3 MB camera with white LED (20X-400X)-YF005, and a UV light lamp (Typ NU-8KL) were used for the experiments.

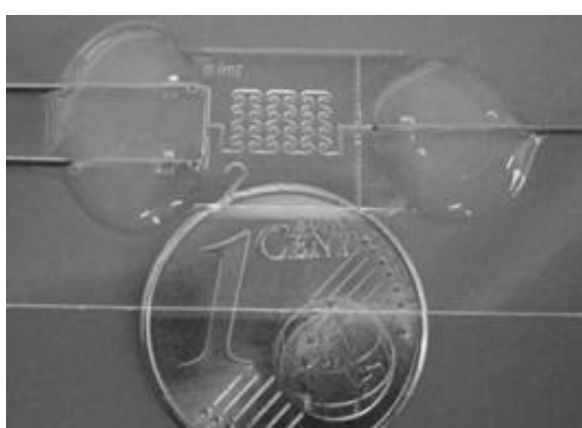

(a)

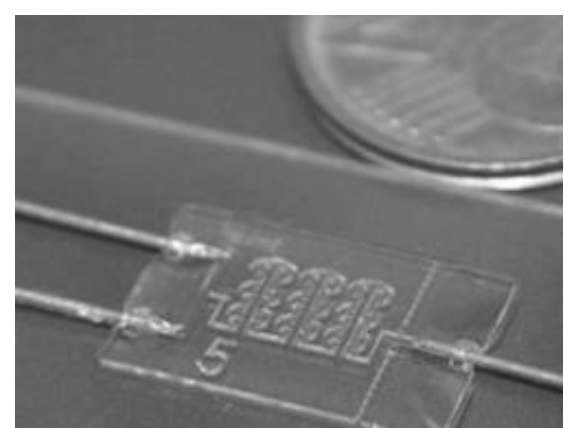

(b)

Figure 8. Pictures of the microfabricated semi-circle (a) and $\Omega(\mathbf{b})$ mixers.

\subsection{Experimental Setup and Results}

Micromixers' performance is examined experimentally by pumping distilled water and fluorescence solution (water with $1 \mathrm{~g} / \mathrm{L}$ of fluorescein isothiocyanate, FITC) at the same mass flow rates using a syringe pump. The flow rates were varied from 3.89 to $19,500 \mu \mathrm{L} / \mathrm{h}$, which correspond to Reynolds numbers from 0.01 to 50 . Transported liquids are exposed to UV light to monitor the fluorescence particle diffusion into DI water. Images and videos of the mixing process are collected using a microscope camera and saved to the computer (Figure 9). 


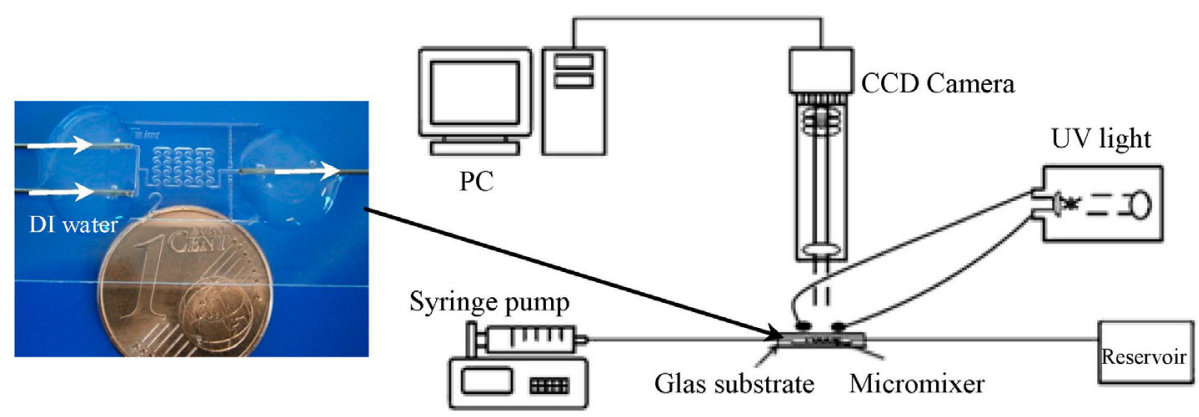

Figure 9. Fabricated micromixer and experimental setup.

\section{Results and Discussion}

The mixing processes in the interlocking semi-circle and $\Omega$ micromixers were experimentally tested and captured images along the micromixers' length were analyzed to quantify the mixing performance. Figure 10 shows different points $(A-G)$ where images of the mixing process at the Reynolds number of 50 were taken.

In Figure 10, it can be observed that the DI water and the fluorescence solution enter the first mixing unit separated as laminar layers moving in parallel flow paths. Due to the curved shape of the first semi-circle and the sudden change in direction, the fluorescence tends to spread radially toward the outer channel wall. However, this does not mean that diffusion occurred but rather that, due to inertial effects (Dean vortices), movement of the particles was initiated. After crossing the first half-circle, the flow direction is deflected by $90^{\circ}$ before entering the second semi-circle. It is very interesting to note that the fluorescence bulk flow shifts its location towards the outer channel wall. This change in flow position through mixing units enhances the diffusion rate and a spreading gradient of fluorescence particles is achieved (Figure 10B-G).

In Figure 10, point $G$, a relatively uniform distribution of the particles is achieved and nearly complete mixing is observed after the last mixing unit. The degree of mixing was quantified by measuring the standard deviation $(\sigma)$ of the intensity distribution of fluorescence particles over each captured image after converting the colors into a grayscale (Equation (2)). The grayscale values $(I)$ of the two fluids are normalized to be 0 for water and 1 for FITC:

$$
\sigma=\sqrt{\frac{1}{N} \sum_{i=1}^{N}\left(I_{i}-\bar{I}\right)^{2}}
$$

where $\bar{I}$ is the gray scale average over all the pixels in the image and $N$ is the number of the pixels in the image. Thus, the value of $\sigma=0.5$ indicates unmixed streams and 0 is for complete mixing.

In Figure 11, the measured fluorescence intensity distribution along the channel width at points A-G is plotted. At point A the difference between the fluorescence intensities along the channel width is very strong. The mean intensity value is 57 a.u. The corresponding standard deviation was calculated along the channel width to be approximately $36 \%$. This large value of the standard deviation indicates that the fluids are not mixed. At point B there is a bimodal distribution of intensity indicative of mixing at the interface as shown. At point $C$ the standard deviation decreases from the average intensity value along the channel width. At point $\mathrm{D}$, the bimodal distribution is further reduced due to increased mixing in the vicinity of the channel center within the elongated interfacial zone. Farther downstream, at points 
$\mathrm{E}$ and $\mathrm{F}$, the gradient of intensity is diminishing even further. At point $\mathrm{G}$, the standard deviation was calculated to be $10 \%$. This $10 \%$ value was considered to be an indication of complete mixing for the rest of the mixer experiments.
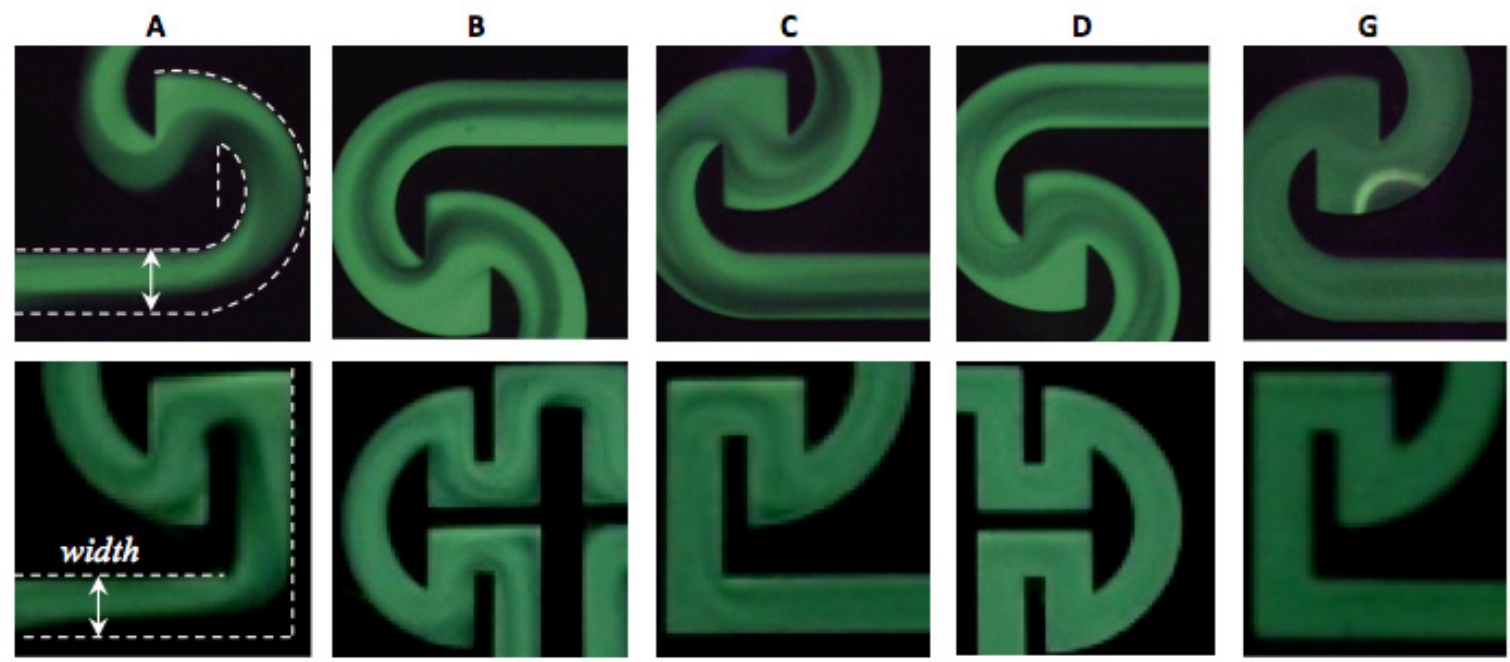

Figure 10. Diffusion process along micromixer channel length of FITC and water at $\operatorname{Re}=50$. Semi-circle shape (2nd row) and $\Omega$ (3rd row).
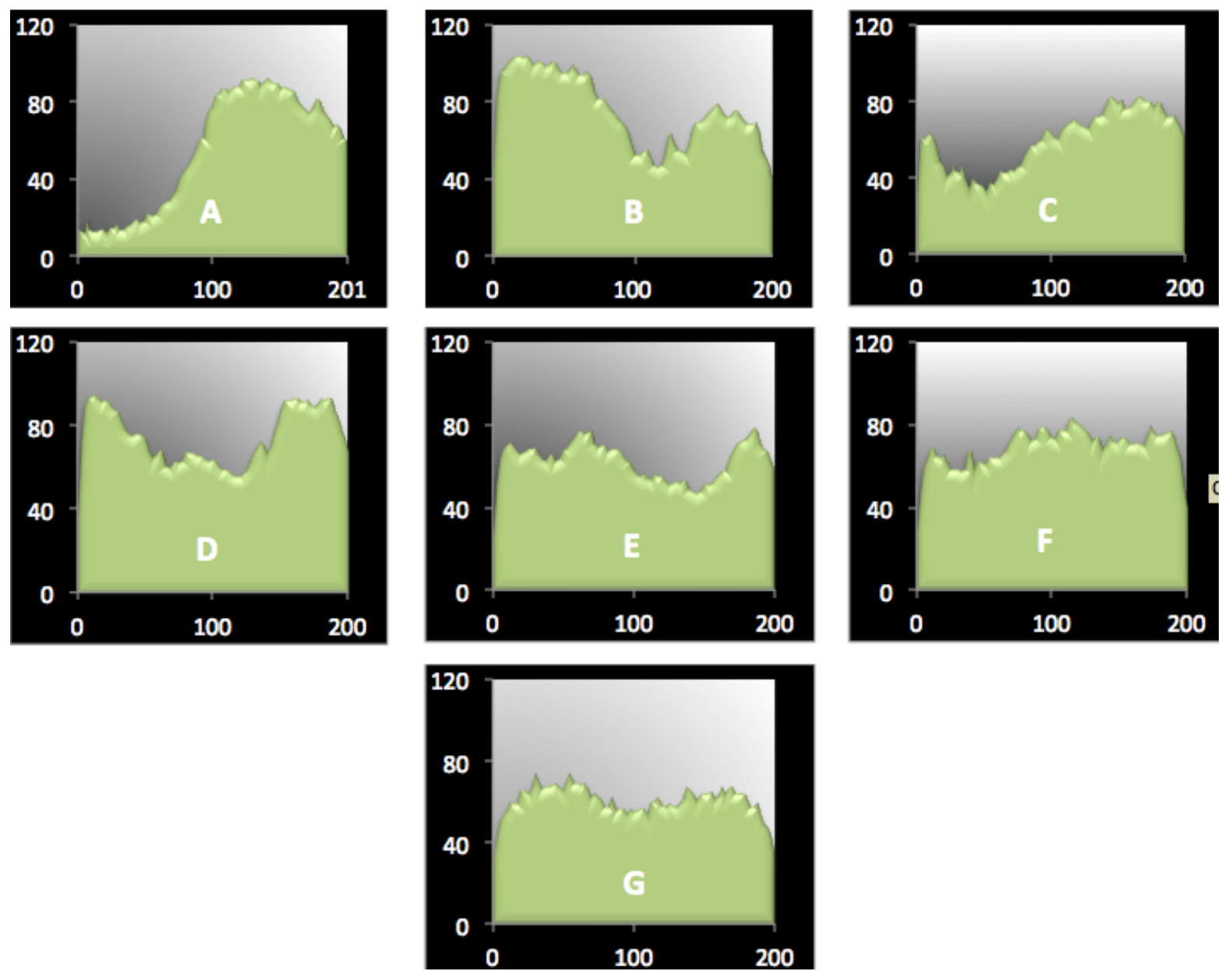

Figure 11. Fluorescence intensity along the channel width at selected points A, B, C, D, E, $\mathrm{F}$, and $\mathrm{G}$ at Re number of 50 . 
Figure 12 presents a comparison between the standard deviation of three micromixer designs for a channel width of $200 \mu \mathrm{m}$ and a wide range of Reynolds numbers.

Figure 13 shows that the experimental and computational model results are in good agreement. It also confirms that the ILSC and $\Omega$ micromixers designed with specified mixing modules are capable of offering relatively complete mixing over the entire range of investigated Reynolds numbers.

A comparison of the experimental results illustrated by the intensity of fluorescence level and the numerical simulation contours of local concentration fraction are shown in Figure 14a,b. A good match is observed between the local concentrations of the first few modules where mixing is taking place. In the last few modules, where mixing is nearly completed, a diminishing of the concentration gradient due to the strong dean flow in the case where the Reynolds number is 50 is recognized.

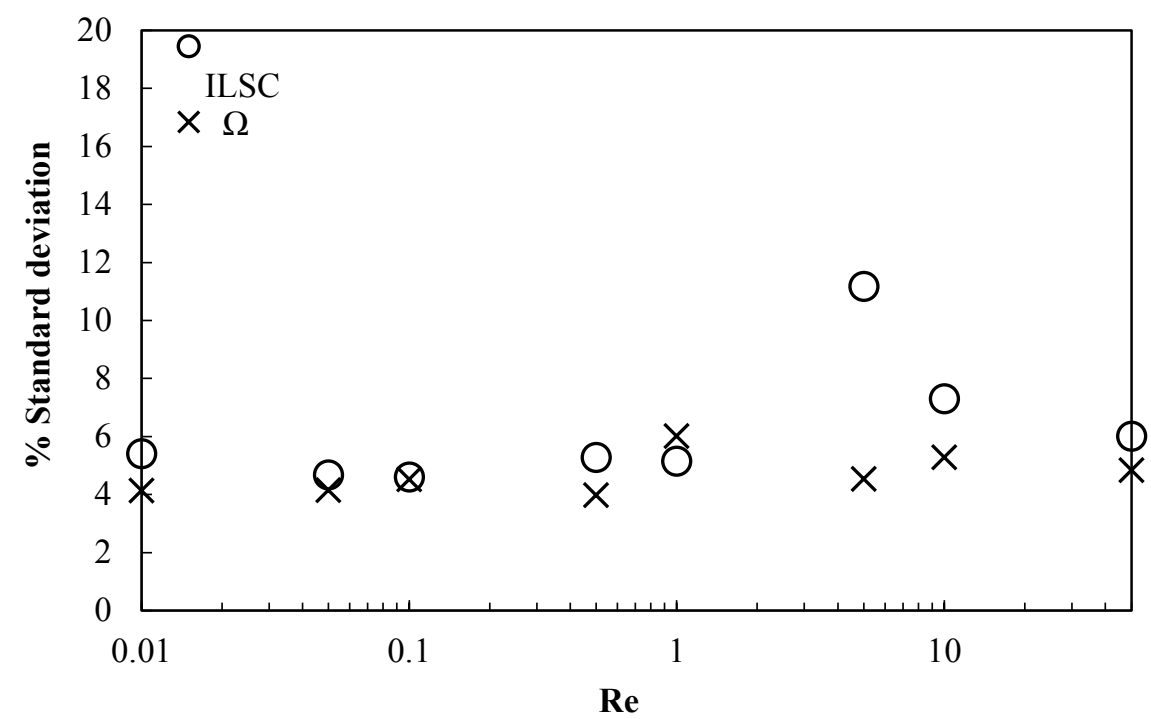

Figure 12. Standard deviation at the channel outlet of the three micromixer designs (200 $\mu \mathrm{m}$ channel width).

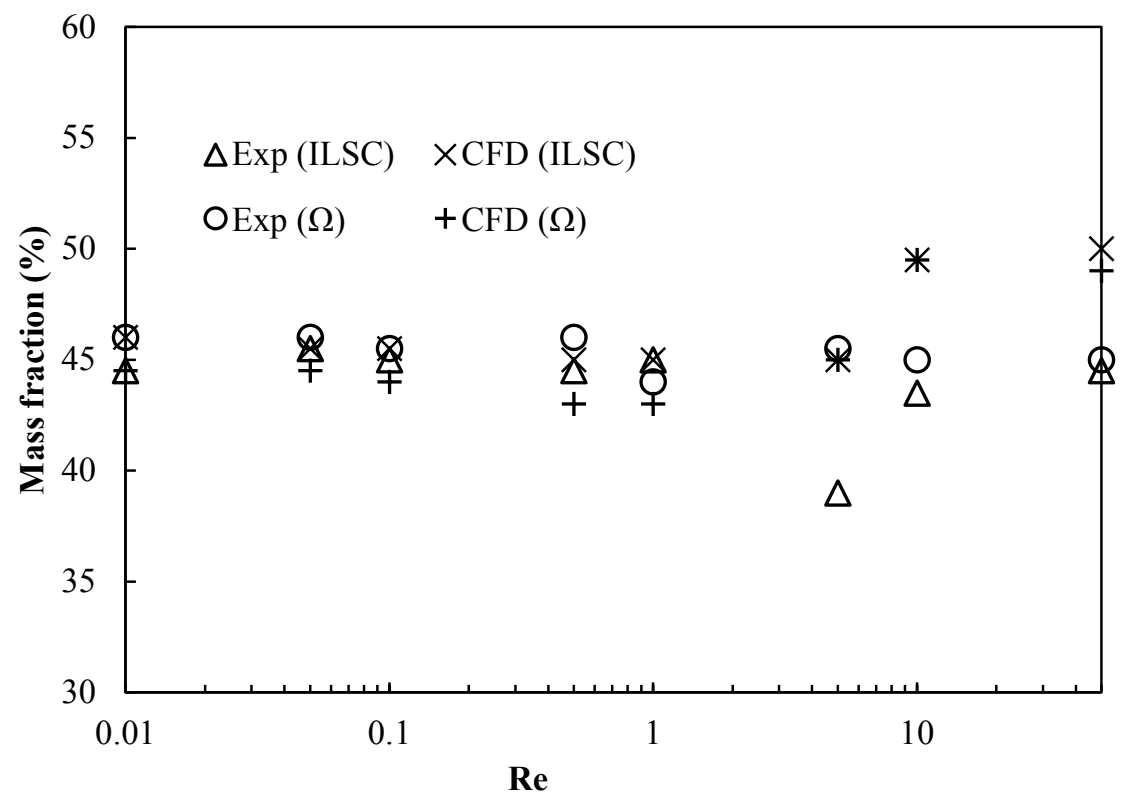

Figure 13. Comparison of mass fraction percentage of experimental and numerical results at channel outlet. 

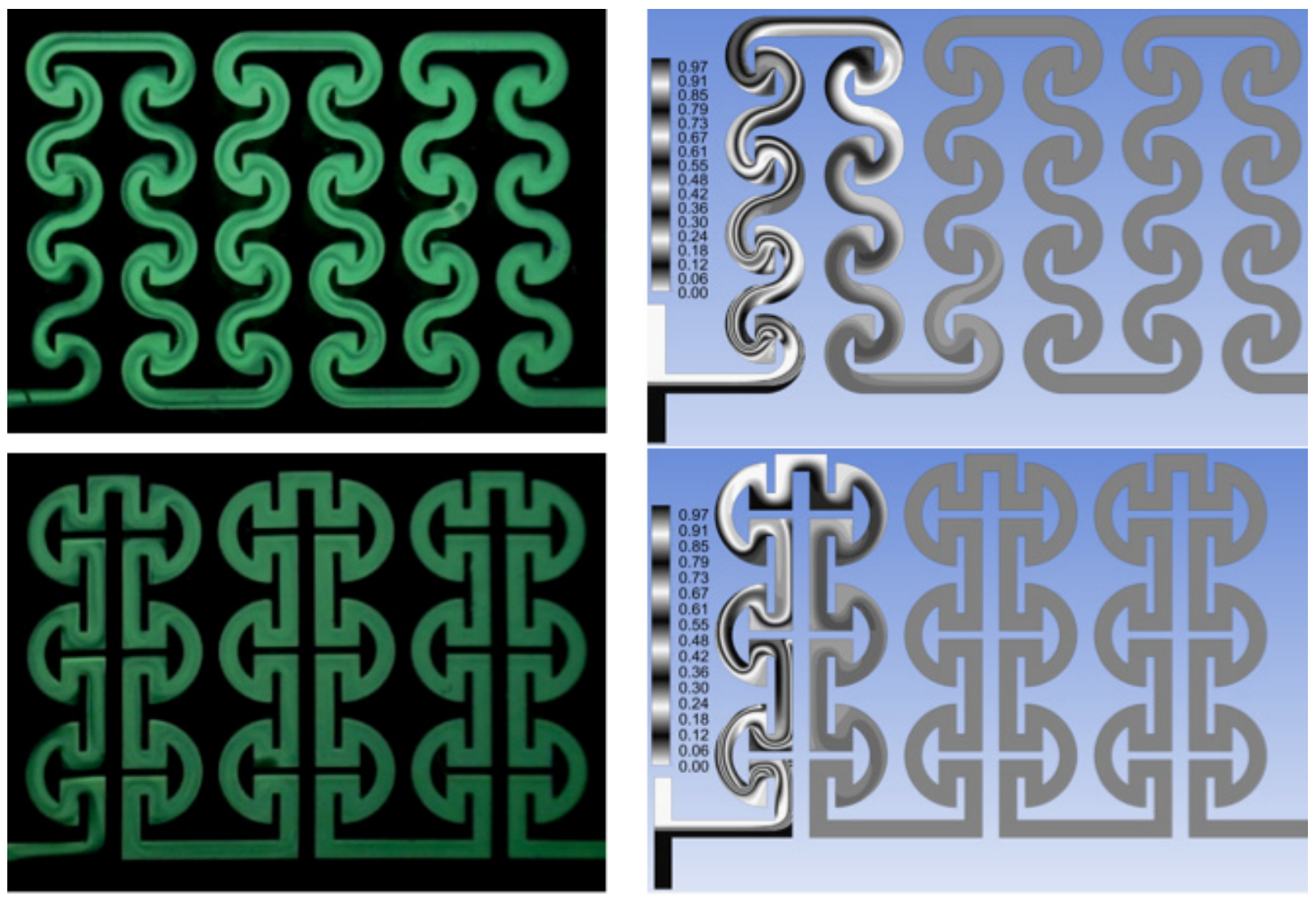

(a)

(b)

Figure 14. Visualization and comparison of mixing results for experiments (a) and numerical contours $(\mathbf{b})$ at $\mathrm{Re}=50$ in $\Omega$ micromixer design.

\section{Conclusions}

This paper introduced new passive micromixer designs that consist of semi-circle shapes arranged in spiral, ILSC, and $\Omega$ configurations. The mixing concept is based on utilizing the combined effect of diffusion and Dean vortices to obtain improved mixing performance. Simulations were used to investigate the new design concepts and experiments were conducted for verification of the computations. The new designs offer:

- Single layer form, which allows easy PDMS fabrication process and passive operation.

- Potential applications in LOC systems working under continuous flow conditions.

- Very promising results of nearly complete mixing in the ILSC and $\Omega$ micromixers over the entire range of Reynolds numbers between 0.01 and 50 and for $\operatorname{Re}>50$ for the spiral micromixer.

Experiments verified the numerical results by analyzing obtained images and estimating the mixing intensity and fluorescence standard deviation for the range of Reynolds numbers.

\section{Acknowledgments}

The authors gratefully acknowledge financial support provided by the German Research Foundation (DFG) within the project "mikroPART-Microsystems for particulate Life-Science-Products". 
One of the authors (Stephanus Büttgenbach) gratefully acknowledges the financial support of the Volkswagen Foundation.

\section{Author Contributions}

Ala'aldeen Al-Halhouli and Mukeet Mohsen designed, simulated, fabricated and characterized the micromixers. Maher Matar contributed to the micromixers design and simulation. Aiman Alshare contributed to the micromixers simulations and generated the proposed mixing correlation. Andreas Dietzel and Stephanus Büttgenbach supervised the project at all stages.

\section{Conflicts of Interest}

The authors declare no conflict of interest.

\section{References}

1. Al-Halhouli, A.T.; Demming, S.; Waldschik, A.; Büttgenbach, S. Implementation of synchronous micromotor in developing integrated microfluidic systems. Micromachines 2014, 5, 442-456.

2. Al-Halhouli, A.T.; Demming, S.; Alahmad, L.; LIobera, A.; Büttgenbach, S. In-line photonic biosensor for monitoring of glucose concentrations. Sensors 2014, 14, 15749-15759.

3. Al-Halhouli, A.T.; Kilani, M.I.; Waldschik, A.; Phataralaoha, A.; Büttgenbach, S. Development and testing of a synchronous micropump based on electroplated coils and microfabricated polymer magnets. J. Micromech. Microeng. 2012, 22, 065027.

4. Nguyen, N.; Wereley, S. Fundamentals and Applications of Microfluidics; Artech House: Norwood, MA, USA, 2006.

5. Nguyen, N.-T.; Wu, Z. Micromixers-A review. J. Micromech. Microeng. 2005, 15, R1.

6. Hessel, V.; Löwe, H.; Schönfeld, F. Micromixers - A review on passive and active mixing principles. Chem. Eng. Sci. 2005, 60, 2479-2501.

7. Tofteberg, T.; Skolimowski, M.; Andreassen, E.; Geschke, O. A novel passive micromixer: Lamination in a planar channel system. Microfluid. Nanofluid. 2010, 8, 209-215.

8. Li, P.; Cogswel, J.; Faghri, M. Design and test of a passive planar labyrinth micromixer for rapid fluid mixing. Sens. Actuators B Chem. 2012, 174, 126-132.

9. Sudarsan, A.; Ugaz, V. Fluid mixing in planar spiral microchannels. Lab Chip 2006, 6, 74-82.

10. $\mathrm{Hu}, \mathrm{Y}$; Chang, M.; Lin, K. A study of two fluids mixing in a helical-type micromixer. Int. Symposium Instrum. Sci. Technol. 2006, 48, 531-536.

11. Liu, R.H.; Stremler, M.A.; Sharp, K.V.; Olsen, M.G.; Santiago, J.G.; Adrian, R.J.; Aref, H.; Beebe, D.J. Passive mixing in a three-dimensional serpentine microchannel. J. Microelectromech. Syst. 2000, 9, 190-197.

12. Hong, C.-C.; Choi, J.-C.; Ahn, C.H. A novel in-plane passive microfluidic mixer with modified Tesla structures. Lab Chip 2004, 4, 109-113.

13. Yang, A.-S.; Chuang, F.-C.; Chen, C.-K.; Lee, M.-H.; Chen, S.-W., Su, T.-L.; Yang, Y.-C. A high-performance micromixer using three-dimensional Tesla structures for bio-applications. Chem. Eng. J. 2015, 263, 444-451. 
14. Doormaal, J.P.; Raithby, G.D. Enhancement of the SIMPLE method for predicting incompressible fluid flows. Numer. Heat Transf. 1984, 7, 147-163.

15. Patankar, S.V. Numerical Heat Transfer and Fluid Flow; Chemical Rubber Company: Boca Raton, FL, USA, 1980.

16. Leonard, B.P. A stable and accurate convective modeling procedure based on quadratic upstream interpolation. Comput. Methods Appl. Mech. Eng. 1979, 19, 59-98.

(C) 2015 by the authors; licensee MDPI, Basel, Switzerland. This article is an open access article distributed under the terms and conditions of the Creative Commons Attribution license (http://creativecommons.org/licenses/by/4.0/). 